Historic, Archive Document

Do not assume content reflects current scientific knowledge, policies, or practices. 



\section{Price List of Field Grown Dahlias}

WILLIAM E. CONGDON 525 South Matlack Street
WEST CHESTER, PA.

Field on Lenape Road, one Block Suuth of Price Street

\section{Decoratives}

Slbert and ............. \$1.00

Alexander Waldie ............. 1.00

$\Lambda$ mun Ra ................... 1.00

Anna Lammey ............... .50

tratia ......................50

Amerien .............25

Bashful Giant ................. 1.00

Beautrice Slocombe ............ 1.00

Ben-1................ 1.00

Bonny Best ................50

Gatherine Cooper ............. .75

Charm ..................... 1.00

Chieftain .................... .50

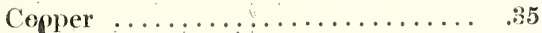

Della Potter ............... .50

Dorothy Robbins ............. .50

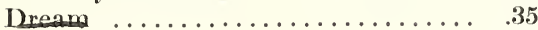

Dr. Tevis ...................50

Earl Williams .............. 1.00

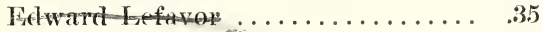

Elizabeth Slocombe .............50

Emperor ................... 1.00

Francis A. ................

Franeis fotote\# .............. 1.00

Glory of New Haven .......... 1.00

Grizzly .................... .75

Hawy Davitson ............... .50

Herbert Slocombe ............ .75

Halvella .................. 1.00

Hochsai .................... .50

Hortulanus Fiet .............. .50

Indian Chief ................ 1.00

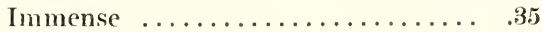

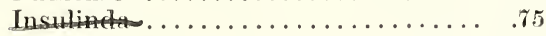

Jim Pollard ................ 1.00

Judge Marean ................ 1.00

Jndge Parker ............... 1.00

King of Autumn ............. .75

King of Commerce ........... .35

King Phillip .................. 1.00

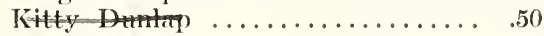

La Toreador .................50

Lady Betty ................ 1.00

I. Grand Maniton ............ .25
L.e Groste .................. 1.00

Mable faft $\ldots \ldots \ldots \ldots \ldots \ldots \ldots \ldots . .50$

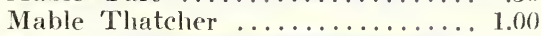

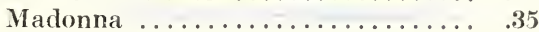

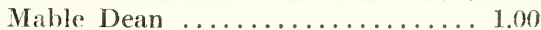

Marie Louise ..................75

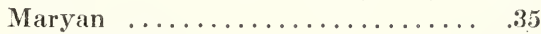

Mephistophles .............. 1.00

Merry Widow ............... .50

Millionaire .................50

Mina Burgle ................25

Mons Le Monard ............. .50

Mrs. Carl Salsbach ............ .75

Mrs. E. C. Boston ............. .50

Mrs. I. D. Y. W mmer .......... .75

Mrs. G. Elkins, Jr. . . . . . . . 1.00

Mrs. Imetey .............. 1.00

Mrs. R. R. Strange ............ .50

Mrs. Scheepers ............... 1.00

Mrs. Thomas Bursh .......... .25

N. C. $4 \ldots \ldots \ldots \ldots \ldots \ldots \ldots \ldots . .50$

Osam Shodow ................. .75

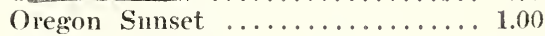

Patrick O'Mara .............. .50

Polaris .................... .50

Paul Michael ................ fis

Pride of California ............. .35

Princess pt.................50

Proxie .................... 1.00

Rosa Nell ...................... .15

San-Mated ................. .50

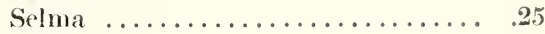

Senarit.................. 1.00

Sitka ......................50

Snowdrift ..................50

Strunner .....................5?

Tenor Alvarez .................35

Theodore Vail ................. .50

W. D'Arey Ryan ...............5

William Slocombe ............ 1.00

Wizard of $\mathrm{Oz} \ldots \ldots \ldots \ldots \ldots \ldots . . .60$

Worlds Wonder ................50

Ysleta ..................... .50 


\section{Cactus}

Ambassador ............... \$1.00

Attraction .................. .50

Ballet Girl ................... 1.00

Chocolate Soldier .............. .75

Cigarette .................. 1.00

Ephriam Gill .................50

Etandard De Lyon ..............35

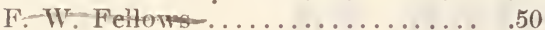

Gee Whiz .................. 1.00

Gladys Sherwood ............. .50

Golden Prince ................ .25

Golden West .................35

Helen Durnbaugh ............. .50

Herbert Raby ............... .50

J. H. Jackson .............. .25

John Riding ............... .50

Justice Bailey ................ .50
Kalif ...................... .50

Mrs. Alfred Stern ............ . $\mathbf{. 3 5}$

Mrs. Estes ................ .50

Mrs. C. Cooper ............... .50

Mrs. Edna Spencer ............ .75

Mrs. Ethel Smith .............. .75

Mrs. Waarnar ............... .35

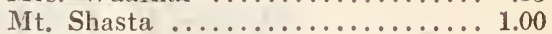

Nerthe ......................35

Nova Pictoris .............. 1.00

Pierott..................... .25

Ruth Gleadell ............... .50

The Eagle ................. .50

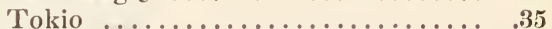

Tom Lundy ......................

Valient ....................50

\section{Paeony}

Billionatre $\ldots \ldots \ldots \ldots \ldots \ldots \ldots \ldots . .50$

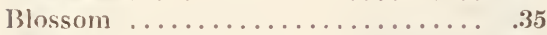

Christine Evers .............. .50

C!e patra ................... .50

Cononchet $\ldots \ldots \ldots \ldots \ldots \ldots \ldots \ldots . .35$

Dr. Perry ...................35

Forest Loma .................35

Geisha ................... .50

He en Iadd Corbett ........... .25

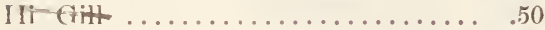

Hulda I)e Jxs ................35

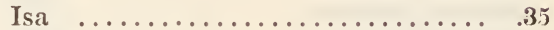

John Slocombe ............. .50

Mary Jean Warner ........... .50

Mondschiebe .................. $\mathbf{. 3 5}$

Natalie Mae ................. .50

Pitti Sing ................. 1.00

Ondawa ....................50

Pearl Ruggles ............. .50

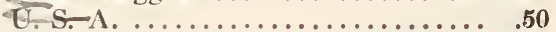

Tan Dyke ...................50

\section{Show}

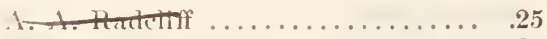

A $\ldots \ldots \ldots \ldots \ldots \ldots . .25$

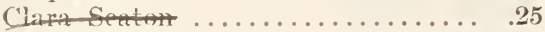

Deelighted ...................25

Golden Opportunity ........... .25
Helen Hollis ................ .25

Mad. Sov. De Gagne ........... 2.25

Maude Adams ................ .25

Mrs. C. D. Anderson ........... .25

Vivian ...................25

\section{Pompons}

All Pompons Are \$.25 Each

Imber Queen

Belle of Springfield

Botoy

Catherine

Dainty

H. C. Winters
Klein Domitea
Imperial
Little Beauty

Little Belle
Little Fred
Madeline
Minnie
Phoebe

Prince Charming

Pure Love

Snowclad

Spy

Zoe

0
5
5
5
5
5
5
5
5
5
5

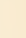

